\title{
Gradhiva
}

GRADHIV

Revue d'anthropologie et d'histoire des arts

$25 \mid 2017$

Gottfried Semper, habiter la couleur

\section{Paul Dumouchel et Luisa Damiano, Vivre avec les robots : essai sur l'empathie artificielle}

Paris, Seuil, coll. « La couleur des idées », 2016

Frédéric Keck

\section{OpenEdition}

Journals

Édition électronique

URL : http://journals.openedition.org/gradhiva/3351

DOI : 10.4000/gradhiva.3351

ISSN : 1760-849X

Éditeur

Musée du quai Branly Jacques Chirac

Édition imprimée

Date de publication : 31 mai 2017

Pagination : 265-266

ISBN : 978-2-35744-095-1

ISSN : 0764-8928

Référence électronique

Frédéric Keck, « Paul Dumouchel et Luisa Damiano, Vivre avec les robots : essai sur l'empathie artificielle », Gradhiva [En ligne], 25 | 2017, mis en ligne le 31 mai 2017, consulté le 24 septembre 2020. URL http://journals.openedition.org/gradhiva/3351 ; DOI : https://doi.org/10.4000/gradhiva.3351

Ce document a été généré automatiquement le 24 septembre 2020.

(c) musée du quai Branly 


\section{Paul Dumouchel et Luisa Damiano,} Vivre avec les robots : essai sur l'empathie artificielle

Paris, Seuil, coll. « La couleur des idées », 2016

\section{Frédéric Keck}

\section{RÉFÉRENCE}

Paul Dumouchel et Luisa Damiano, Vivre avec les robots : essai sur l'empathie artificielle.

Paris, Seuil, coll. « La couleur des idées », 2016, 240 p. 


\title{
Paul Dumouchel
}

Luisa Damiano

\author{
VIVRE \\ AVEC LES ROBOTS \\ Essai sur l'empathie artificielle
}

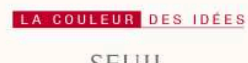

SEUIL

1 Cet ouvrage est une contribution importante à l'anthropologie des robots, domaine en pleine expansion, à partir de la philosophie cognitive et sociale. Paul Dumouchel et Luisa Damiano travaillent tous deux avec des roboticiens japonais, mais à la différence de Joffrey Becker, Emmanuel Grimaud ou Denis Vidal ${ }^{1}$, ils ne proposent pas ici une enquête ethnographique sur les robots. Leur propos est plutôt d'interroger les fondements anthropologiques que l'ethnographie des robots vient mettre en question. À ce titre, ils prolongent et approfondissent ce courant encore minoritaire pour le faire converger avec le grand fleuve de l'anthropologie générale.

2 L'ouvrage commence par rappeler l'étymologie du terme "robot» forgé par Karel Capek en 1920 dans une pièce de théâtre pour désigner des androïdes faits de matériaux biologiques synthétiques. Le terme tchèque "robota "signifiant "corvée " ou «esclave ", le problème des robots a longtemps consisté à savoir s'ils pourraient remplacer les humains dans leurs tâches les plus ingrates. Le progrès des robots réels (et non fictionnels) depuis une trentaine d'années mais surtout la place qu'ils ont prise dans les sociétés industrialisées, notamment au Japon, obligent à poser une autre question : quelles relations pouvons-nous attendre de nos interactions avec les robots et que révèlent-elles des modalités interactionnelles en général? "Qu'est-ce que la robotique sociale nous apprend au sujet de qui nous sommes et de notre vivre ensemble?» (p. 27)

3 Le sous-titre de l'ouvrage est d'emblée provocateur. Il ne s'agit pas de considérer les robots comme des « intelligences artificielles " pour s'interroger jusqu'à quel degré ils peuvent imiter, voire améliorer, l'intelligence humaine. En parlant d'« empathie artificielle", Dumouchel et Damiano proposent d'étudier les émotions que nous pouvons attribuer aux robots et les dynamiques interactionnelles qu'elles enclenchent: «La dynamique des échanges affectifs qui s'effectuent par le moyen de l'expression des 
affects constitue un mécanisme intraspécifique de coordinations et de déterminations réciproques de nos moyens d'action. » (p. 27)

4 Pour attribuer des émotions aux robots (amour, joie, tristesse, honte...), il faut une définition plus stricte de la notion que celle que son expansion actuelle laisse flotter. Il ne s'agit nullement des robots ménagers, industriels ou financiers qui broient nos aliments, construisent nos voitures ou autorisent nos crédits, mais plutôt de ceux conçus par les roboticiens pour tester les réactions du public. Ces robots, à la suite du texte visionnaire de Masahiro Mori publié pour la première fois en anglais en 1970 sous le titre The Uncanny Valley ${ }^{2}$, visent en effet à jouer sur le trouble relationnel que suscite un artefact anthropomorphe. Certains, relevant de la "robotique sociale externe", réagissent aux comportements humains par des mouvements apparemment signifiants (Keepon, Paro, NAO, KASPAR, Saya, Face, Geminoid : voir tableau p. 128); d'autres sont programmés pour exprimer et reconnaître des émotions de manière interne (Barthoc, Maggie, Sage et Kismet - on pourrait ajouter Berenson, le robot expérimenté au musée $\mathrm{du}$ quai Branly pour reconnaître des émotions esthétiques ${ }^{3}$ ). Dumouchel et Damiano proposent de caractériser ces robots comme des «substituts» au sens de "quelqu'un qui remplace quelqu'un d'autre sans lui prendre sa place, c'est-à-dire sans lui enlever sa fonction» (p.39). Le substitut est donc doté, outre d'une fonctionnalité, d'une présence, d'une autorité et d'une autonomie.

5 Cette définition permet à Dumouchel et Damiano de distinguer deux tendances dominantes. L'une, inspirée de la sociologie des sciences de Bruno Latour ${ }^{4}$, considère le robot comme un acteur dans une chaîne technique et non comme une personne engagée dans des rapports sociaux (p. 55). L'autre, inspirée de l'éthique des robots militaires d'Armin Krishnan ${ }^{5}$, les considère comme des machines prenant des décisions sur la vie et la mort des individus (p. 195). Ces deux courants ont en commun de ne pas attribuer aux robots des émotions, et donc de manquer l'incertitude interactionnelle entre les humains et les robots et la distribution cognitive qui s'effectue entre les partenaires de l'interaction. Dumouchel et Damiano proposent une «éthique synthétique » (p. 223) pour décrire ces interactions entre des "agents analytiques » (p. 215).

6 Une telle approche cesse de décrire les robots comme des esclaves ou des soldats pour les présenter plutôt comme des anthropologues expérimentant les modalités d'interaction sociale en terrain inconnu (p. 65). L'anthropologie des robots n'est pas seulement une anthropologie réflexive, le roboticien construisant avec son robot un miroir de l'anthropologue lorsqu'il pense les difficultés qu'éprouve son corps à comprendre de nouvelles modalités d'interaction sociale. C'est aussi une anthropologie politique, pour autant que l'entrée des robots dans les sociétés contemporaines pose à nouveau les questions de justice distributive qui sont au fondement de ces sociétés. La question, en l'espèce, est de savoir si les humains traiteront les robots comme des machines vite envoyées à la casse - «Politiquement un robot ne vaut rien. On peut en sacrifier autant qu'on veut!» (p. 204) - ou s'ils pourront nouer avec eux des rapports sociaux réciproques et équitables. Le problème des valeurs que nous pouvons construire avec les robots à partir des émotions que nous partageons avec eux reste donc ouvert à l'issue de ce livre fondamental. 


\section{NOTES}

1. Voir Emmanuel Grimaud, Dieux et robots: les théâtres d'automates divins de Bombay. Apt, L'Archange Minotaure, 2008 ; Emmanuel Grimaud et Zaven Paré, Le Jour où les robots mangeront des pommes: conversations avec un Geminoid. Paris, Éditions Petra, 2011; Denis Vidal et Emmanuel Grimaud (dir.), Gradhiva 15, Robots étrangement humains, 2012; Joffrey Becker, Humanoïdes: expérimentations croisées entre arts et sciences. Nanterre, Presses universitaires de Paris Ouest, 2015 ; Denis Vidal, Aux frontières de l'humain: dieux, figures de cire, robots et autres artefacts. Paris, Alma, 2016.

2. Masahiro Mori, «La vallée de l'étrange », trad. d'Isabel Yaya, Gradhiva 15, 2012 : 26-33.

3. Voir Denis Vidal et Philippe Gaussier, «Un robot comme personne. Ontologies comparée et expérimentale au musée du quai Branly », Terrain 62, 2014 : 152-165.

4. Bruno Latour, Petites leçons de sociologie des sciences. Paris, La Découverte, 2006.

5. Armin Krishnan, Killer Robots: the Legality and Ethicality of Autonomous Weapons. Burlington, Asgate Publishing Company, 2009.

\section{AUTEURS}

\section{FRÉDÉRIC KECK}

Frederic.KECK@quaibranly.fr 